\title{
EDITORIAL
}

\section{Advancing the Field through Multiple Media}

Recent years have seen a dramatic shift in the way information is delivered and accessed by people in the U.S. and around the globe. Traditional print media are being steadily replaced by digital media in almost all areas of human endeavor, from online news to electronic book readers. As researchers, educators, and scientists our community is at the forefront of innovation and adoption of technologies for sharing information and collaborating with diverse communities.

For a journal like JRST, the move from print media to digital media provides enormous opportunities. Electronic distribution provides greater access to the journal globally. Digital communication allows the work of our authors to be accessed by wider audiences, and provides the opportunity for us to introduce the work of our research community to a broader range of intellectual, policy, and practice communities. This past year, JRST experienced 140,000 accesses through Google alone. Digital storage and archiving is providing journals in the sciences with the ability to include a wide range of supplementary and supporting information with articles that further inform and drive follow-up work in the field. Like many journals in the sciences, we need to offer the possibility of including richer and deeper content—photos, video, data sets, instruments, relevant transcripts and artifacts-that reflect the rich and evolving nature of research in science education. Significantly, electronic distribution is providing these and other benefits while improving our sustainability, reducing our impacts on the environment, and increasing our impact on our field.

Below we share with you several ways in which we are working towards greater presence in the field and globally while helping to sustain our environment by utilizing emerging digital technologies and multiple media.

We are working closely with our colleagues at Wiley-Blackwell on a major overhaul of the JRST website, which we hope will be introduced to the community during the first months of 2011 . The upgrade to the Wiley-Blackwell electronic publishing systems allows us to make the online presence of the journal a more feature-rich and dynamic experience. It will also incorporate a broad range of additional functionality when viewing journal articles and related content in online and electronic media.

We will also introduce the first "virtual issue" of the journal in late spring. Our virtual issues will unite articles with similar topics from past issues of JRST that inform an aspect of the field, and will be marketed to introduce JRST and the work of our author community to broader audiences. The first virtual issue will be edited by Associate Editor Julie Luft in collaboration with the National Science Teachers Association's Research Committee, and will be targeted to provide a collection of articles from JRST that are particularly relevant to the practitioner community. Collaborating with our publishers, we will make this virtual issue widely available to members of the National Science Teachers Association and other practitioner communities.

To support the enhanced online presence of the journal, we are now accepting supplementary materials as part of manuscript submissions, which readers can access when an article is viewed or retrieved online. Supplementary material may include traditional appendices, additional tables, instruments, transcripts, etc. which are relevant to support the claims in the paper but which may not be appropriate by virtue of length or type of media for inclusion in the print article. We are also exploring the opportunities new technologies provide for data sharing, including media files, data sets, and lengthier instruments which are of service to the

Correspondence to: Angela Calabrese Barton, Joseph Krajcik, and Robert Geier; E-mail: jrst@msu.edu DOI 10.1002/tea.20376

Published online 15 March 2010 in Wiley InterScience (www.interscience.wiley.com). 
community. We encourage authors to submit supplementary materials along with their article as they see fit. As part of the review process, if the editorial team notes material in a manuscript that would be better placed in the supplementary materials, we will make that recommendation to the authors. Our newly updated guide to authors contains a number of important changes in how we accept manuscripts, and offers specific details and instructions on the submission of supplementary materials.

One of the challenges of electronic media is the need to make content available rapidly to have the impact we desire. To stay on the forefront of our field and to provide the service our author community demands, JRST must provide timely publication so that important work can reach our diverse stakeholders when it is most relevant. The recent editors of JRST and their team made enormous strides in bringing JRST online and in reducing a publication delay that had reached nearly two and a half years. We are committed to continuing that effort with your help, so that our authors can rely on JRST as a place to submit important work for the most timely publication. The online supplemental materials will help in reducing the print page count and thereby improving the publication time for all contributors. However, supplemental materials are not a panacea. JRST authors need to pay careful attention to page count, and to work diligently to provide concise presentations and arguments. Tables presented in article bodies should be highly relevant, while we encourage background tables and materials be placed in the supplementary materials section. As editors, we will work with authors in the review and pre-press stages to make optimum use of our multiple media. Together, our attention to these issues as researchers and writers will further reduce our print delay and provide all of our authors with more timely and powerful publication.

We are excited to have your support as we lead the journal through the next 5 years, and we are delighted to help move the journal toward new standards and practices made possible with advancing technologies. As with any new endeavor, we know problems and glitches will surface, and we welcome your comments and suggestions.

Angie, Joe, \& Bob

Angela Calabrese Barton, Joseph Krajcik, and Bob Geier Editorial Office, Journal of Research in Science Teaching Michigan State University and University of Michigan jrst@msu.edu 